\title{
The contributions of metal impurities and tube structure to the toxicity of carbon nanotube materials
}

\author{
Cuicui $\mathrm{Ge}^{1}$, Yang $\mathrm{Li}^{1}$, Jun-Jie Yin ${ }^{2}$, Ying Liu ${ }^{1}$, Liming Wang ${ }^{1}$, Yuliang Zhao ${ }^{1}$ and Chunying Chen ${ }^{1}$
}

Due to the existence of considerable quantities of metallic and carbonaceous impurities, the key factor and mechanism for the reported toxicity of carbon nanotubes (CNTs) are unclear. Here, we first quantify the contribution of metal residues and fiber structure to the toxicity of CNTs. Significant quantities of metal particles could be mobilized from CNTs into surrounding fluids, depending on the properties and constituents of the biological microenvironment, as well as the properties of metal particles. Furthermore, electron spin resonance measurements confirm that hydroxyl radicals can be generated by both CNTs containing metal impurities and acid-leachable metals from CNTs. Several biomolecules facilitate the generation of free radicals, which might be due to the participation of these biomolecules in redox cycling influenced by $\mathrm{pH}$. Among several major metal residues, Fe has a critical role in generating hydroxyl radicals, reducing cell viability and promoting intracellular reactive oxidative species. Cell viability is highly dependent on the amount of metal residues and iron in particular, but not tube structure, while the negative effect of CNTs themselves on cell viability is very limited in a certain concentration range below $80 \mu \mathrm{g} \mathrm{ml}^{-1}$. It is crucial to systematically understand how these exogenous and endogenous factors influence the toxicity of CNTs to avoid their undesirable toxicity.

NPG Asia Materials (2012) 4, e32; doi:10.1038/am.2012.60; published online 7 December 2012

Keywords: biological microenvironments; carbon nanotube; metal impurities; reactive oxygen species; toxicity

\section{INTRODUCTION}

Engineered nanomaterials possess novel properties and have been used worldwide in numerous consumer products, for example, food, clothes, pharmaceuticals and cleaning products. Estimates suggest that by 2015 nanotechnology will have a trillion-plus dollar global economic impact. ${ }^{1}$ Therefore, ensuring the safety of nanomaterials is of great importance to the tremendous commercial applications of nanotechnology. Safety evaluation of nanomaterials should consider their behaviors in various aspects, including their interaction with proteins, DNA, lipids, membranes, organelles, cells, tissues, biological fluids and even the dissolution of metal constituents. ${ }^{2-4}$

Carbon nanotubes (CNTs) have attracted great interest from both scientists and the industry because their high aspect ratios, strength and remarkable physical properties make them a unique material with a wide range of promising applications. Many applications of CNTs in biomedicine have been proposed, including nanoelectronics, ${ }^{4}$ biosensors, ${ }^{5}$ biomolecular recognition devices and molecular transporters, ${ }^{6}$ artificial water channels, ${ }^{7}$ cancer therapy ${ }^{8,9}$ and photoacoustic molecular imaging. ${ }^{10}$ Because transitional metals are often used as catalysts during CNT synthesis, ${ }^{11}$ it is unavoidable that as-received CNTs are contaminated by catalyst residues. ${ }^{12}$ The catalyst precursor (such as iron carbonyl) decomposes, and nanometer-sized metal particles form from the decomposition products. Therefore, removing the metallic catalyst (typically $\mathrm{Fe}$, $\mathrm{Co}$ and/or $\mathrm{Ni}$ ) used in the growth of CNTs and other carbonaceous components is one of the fundamental challenges for the application of CNTs in many fields. ${ }^{13}$ Many types of purification methods have been developed, including chemical, physical and chromatographic purification. ${ }^{14,15}$ Because some of the metallic particles are protected by a graphitic shell, rigorous purification procedures might damage the tube structure of CNTs. Therefore, metal catalyst residues frequently remain, even after complicated purification steps. ${ }^{16}$

It has been proposed that the presence of metal impurities might significantly influence the properties of CNTs and the behavior of devices built from these materials. Iron oxide nanoparticles (NPs) have been studied extensively as magnetic resonance imaging contrast agents. Several groups reported the use of metal impurities, such as iron oxides, in CNTs for magnetic resonance imaging. Choi et al. ${ }^{17}$ first demonstrated the use of the SWNT (single-walled nanotube)/ iron oxide NP complexes as multimodal biomedical imaging agents. In another study, the presence of single-walled CNTs (SWCNTs) with associated metal impurities was detected in vivo using noninvasive MR techniques. ${ }^{18}$ In a recent study, the metallic catalyst NPs attached to nanotubes were utilized as the T2-contrast agent in magnetic resonance imaging of SWNT-labeled human mesenchymal stem cells. ${ }^{19}$

\footnotetext{
${ }^{1}$ CAS Key Lab for Biomedical Effects of Nanomaterials and Nanosafety, National Center for Nanoscience and Technology of China and Institute of High Energy Physics, Chinese Academy of Sciences, Beijing, China and ${ }^{2}$ Center for Food Safety and Applied Nutrition, Food and Drug Administration, College Park, Washington DC, MD, USA

Correspondence: Professor Y Zhao or Professor C Chen, CAS Key Lab for Biomedical Effects of Nanomaterials and Nanosafety, National Center for Nanoscience and Technology of China and Institute of High Energy Physics, Chinese Academy of Sciences, 11 Beiyijie, Zhongguancun, Beijing 100190, China. E-mail: zhaoyuliang@ihep.ac.cn or chenchy@nanoctr.cn

Received 20 July 2012; revised 20 October 2012; accepted 22 October 2012
} 
However, the presence of metal impurities can cause confusion about the biocompatibility, toxicity and health risks of CNTs. ${ }^{3,20}$ For example, intracellular reactive oxygen species (ROS) were reported to be dependent on contaminants ${ }^{21}$; however, another report showed that neither purified nor non-purified SWCNTs containing significant amounts of iron were able to generate the intracellular production of superoxide radicals or nitric oxide in RAW 264.7 macrophages. $^{22}$ Ultrafine, transition-metal particles pose documented health risks upon inhalation, but their contributions to the toxicity of CNTs remain unclear due to their apparent encapsulation and immobilization by carbon shells. ${ }^{23}$ To our knowledge, the issue is due to the biologically available degree of residual metal impurities. Despite their apparent encapsulation, toxicologically significant quantities of catalyst residues are released from purified CNTs supplied by commercial vendors when the CNTs interact with the surrounding physical fluid. ${ }^{24,25}$ Biological systems are generally able to integrate multiple pathways of toxicity into a limited number of pathological outcomes. Due to their small size, large surface area and high reactivity, the particle-induced production of ROS and oxidative injury has become an established paradigm for NP toxicity. ${ }^{26,27}$ Another paradigm of NP toxicity is the release of toxic ions when the thermodynamic properties of a material (including surface-free energy) favor particle dissolution in a suspending medium or biological environment. ${ }^{27,28}$ Therefore, it is important to quantify the dissolution of metal residues from CNTs into biological fluids to estimate the role of dissolved metal ions contributing to cellular toxicity.

Potential routes of nanomaterial exposure include the gastrointestinal tract, skin, lungs and systemic administration for diagnostic and therapeutic purposes. ${ }^{29,30}$ Nanomaterials interact with different types of cells, body fluids and proteins, which have a vital role in their biological effects and distribution throughout the body. Therefore, we aim to investigate the dynamic dissolution of metal residues in CNTs upon interaction with several, typical biological fluids, including artificial gastric fluid (AGF) and artificial intestinal fluid (AIF), mildly acidic conditions (at typical lysosomal $\mathrm{pH}$ of 4.5), artificial lung fluid (Gamble's solution, extracellular pH, near 7) and RPMI 1640 cell culture media (RPMI contains more than 40 components). In a cellfree model, the generation of free radicals by CNTs and metal residues was studied, and biologically relevant reducing agents were added to more closely mimic the cellular milieu. Finally, their effect on cellular viability by both CNTs and metal residues was measured, and the possible mechanism was investigated.

\section{MATERIALS AND METHODS}

\section{Chemicals and NPs}

SWCNTs were provided from Shenzhen Nanotechnologies Co., Ltd. (Shenzhen, China) and Helix Material Solutions, Inc. (Richardson, TX, USA). All reagents were of analytical purity or greater and were obtained from the Beijing Institute of Chemical Reagents, Sigma-Aldrich Corporation (St. Louis, MO, USA) and Invitrogen Co. (Molecular Probes, Eugene, OR, USA). The QC-21 quality control standard solution and indium standard solution were obtained from SPEX Plasma Standard (SPEX, Mentuchen, NJ, USA). Stock standard solutions were obtained at a concentration of $1000 \mathrm{mgl}^{-1}$ of each metal (As, Be, Ca, Cd, Co, Cr, Cu, Fe, Li, Mg, Mn, Mo, Ni, Pb, Sb, Se, Sr, Ti, Tl, V, Zn; GBW 08617, National Research Center for CRMs, China). The working standards were diluted daily as required.

\section{Procedure for incubation and inductively coupled-plasma mass spectrometry measurements}

SWCNT suspensions $\left(1.5 \mathrm{mg} \mathrm{ml}^{-1}\right)$ were prepared by dispersing the CNT powder in artificial fluids. The obtained suspensions were incubated at $37^{\circ} \mathrm{C}$ with stirring (100 r.p.m.). After settling, $1 \mathrm{ml}$ of the supernatant was removed and replaced with an equal volume of artificial fluid added to the suspension. In all, $1 \mathrm{ml}$ of supernatant was transferred to centrifuge tubes and centrifuged twice for $15 \mathrm{~min}$ at $9000 \mathrm{~g}$ to remove any remaining particles. The obtained leachates were analyzed via inductively coupled-plasma mass spectrometry using a Thermo Elemental X7 spectrometer (Thermo-Fisher Scientific, Winsford, UK).

\section{Electron spin resonance (ESR) measurements}

ESR spectroscopy in conjunction with a spin-trapping technique was used to examine the free radical-generation capability of leached metals. All ESR measurements were carried out at ambient temperature using a Bruker EMX ESR Spectrometer (Bruker Biospin, Billerica, MA, USA). Each experiment was repeated at least three times. All ESR measurements were carried out using the following settings to detect the spin adduct between DMPO (5,5-dimethylpyrroline $\mathrm{N}$-oxide) and hydroxyl radical: $10 \mathrm{~mW}$ microwave power, $100 \mathrm{G}$ scan range and $1 \mathrm{G}$ field modulation.

The reducing agents used were glutathione and cysteine (to mimic enzymes containing a thiol active site), NADPH and NADH ( $\beta$-nicotinamide adenine dinucleotide $2^{\prime}$-phosphate reduced tetrasodium salt hydrate and $\beta$-Nicotinamide adenine dinucleotide, reduced disodium salt hydrate, respectively, to mimic biologically relevant reducing equivalents) and ascorbic acid (a classical antioxidant). The generation of free radicals under these conditions was studied to better understand the effects of physiologically relevant reducing agents on the generation of free radicals and potential toxicity. Free-radical generation was examined under conditions mimicking three different physiological environments, that is, AGF, artificial lysosomal fluid (ALF) and AIF ( $\mathrm{pH}$ $1.2,4.0$ and 6.8 , respectively). The final concentrations of all reagents in the reaction mixture were thus: $0.5 \mathrm{mg} \mathrm{ml}^{-1} \mathrm{CNT}, 50 \mathrm{~mm}$ buffer, $0.25 \mathrm{~mm}$ reducing agent, and $0.1 \mathrm{~mm}$ hydrogen peroxide. DMPO was used as the spin trap. ESR spectra were recorded after 1 and $10 \mathrm{~min}$ of reaction initiation.

\section{Cell viability assay}

The cellular viability assay, involving the reduction of a water-soluble tetrazolium salt, was performed using a Cell Counting-8 Kit (Dojindo Laboratories, Kumamoto, Japan) according to the instructions of the manufacturer. An aliquot of $100 \mu \mathrm{l}$ of a cell suspension (10000 cells per well) was seeded into a 96-well plate and incubated in a humidified incubator $\left(\right.$ at $37^{\circ} \mathrm{C}$, $\left.5 \% \mathrm{CO}_{2}\right)$. After $24 \mathrm{~h}$, the medium was replaced with fresh medium containing c-leachates or CNTs at different concentrations. After $24 \mathrm{~h}$, the culture supernatant was removed, and the cells were washed three times with phosphate-buffered saline (PBS). Then, a mixture of one volume of the tetrazolium reagent from the Cell Counting Kit- 8 and 10 volumes of medium was added to the cells. After $2 \mathrm{~h}$, the absorbance was measured at $450 \mathrm{~nm}$ using a microplate reader (Bio-Rad 680, Hercules, CA, USA) detection: $450 \mathrm{~nm}$, reference: $650 \mathrm{~nm}$ ) to test the cell viability. Each experiment was repeated independently three times.

\section{Measurement of intracellular ROS and superoxide dismutase (SOD)}

Cells (density $4 \times 10^{5} \mathrm{ml}^{-1}$ ) were seeded into a 6-well plate for flow cytometry and another culture plate with glass coverslips for laser confocal scanning microscopy. After $24 \mathrm{~h}$, the medium was replaced with fresh medium containing c-leachates at different concentrations. After incubation for $24 \mathrm{~h}$, the cells were washed 2-3 times with PBS, and CM- $\mathrm{H}_{2} \mathrm{DCFDA}$ (5-(and-6)chloromethyl-2', $7^{\prime}$-dichlorodihydrofluorescein diacetate, acetyl ester) was added into each well. After incubation for $30 \mathrm{~min}$, the cells were washed three times with PBS. Then, the cells were tested by two different methods; flow cytometry ( $488 \mathrm{~nm}, \mathrm{FL} 1)$ was used to determine the fluorescence intensity in cells, and the cells were also viewed under a laser confocal scanning microscope (Olympus FV 500, Tokyo, Japan).

An SOD Assay Kit (SOD Assay Kit-WST; Dojindo Molecular Technologies, Gaithersburg, MD, USA) was used to determine the SOD activity in cells. 



d

\begin{tabular}{ccc}
\hline Sample & Main metal residues (wt\%) & $\begin{array}{c}\text { Total metal } \\
\text { (wt } \%)\end{array}$ \\
\hline CNT-1 & Fe 0.7\%, Cr 1.1\%, Mo 0.3\% & $2.10 \%$ \\
CNT-2 & Fe 2\%, Mo 0.6\%, Ni 0.1\%, Cr 0.1\% & $2.80 \%$ \\
CNT-3 & Fe 9.3\%, Co 4.8\%, Mo 1.2\% & $15.30 \%$ \\
CNT-4 & Co 0.4\%, Y 3.3\%, Ni 11\% & $14.70 \%$ \\
\hline
\end{tabular}

Figure 1 (a-c) High-resolution transmission electron microscopic images of carbon nanotube (CNT)-1 (a), CNT-3 (b) and metal residues in CNTs (c). (d) The content of main metal residues contained in CNTs determined using inductively coupled-plasma mass spectrometry (unit: $\mu g g^{-1}$ ).

\section{F-actin fluorescent staining}

Cells were cultured to confluence on glass coverslips in medium containing c-leachate (AGF) or $10 \mu \mathrm{g} \mathrm{ml}^{-1} \mathrm{CNT}-2$. After $24 \mathrm{~h}$, the cells were washed three times with PBS and fixed in $2.5 \%$ glutaraldehyde in PBS at room temperature for $5 \mathrm{~min}$ and $4 \mathrm{~h}$ at $4{ }^{\circ} \mathrm{C}$. Subsequently, the cells were washed three times with PBS and permeabilized for 5 min with $0.2 \%$ Triton X-100 in PBS. The cells were then washed three times with PBS. Actin was visualized using rhodaminelabeled phalloidin ( $1 \mu \mathrm{M}$ in PBS with $0.1 \%$ bovine serum albumin) for approximately $30-40 \mathrm{~min}$. After the cover slips were washed three times with PBS, the cells were viewed under a laser confocal scanning microscope (Olympus FV 500).

\section{RESULTS AND DISCUSSION}

\section{Characterization of CNTs}

The HRTEM (high-resolution transmission electron microscopy) characterization of CNTs was performed using a Tecnai G2 F20 U-TWIN transmission electron microscope (FEI Company, Hillsboro, OR, USA). HRTEM images showed the significant difference of the metal residues between CNT-1 and CNT-3 (Figure 1a and b). As shown in Figure 1c, it is clear that metal residues are encapsulated by the carbon shell. Inductively coupled-plasma mass spectrometry was used to provide a quantitative analysis of metal residues using a previously published method. ${ }^{31}$ There are clear differences in the metal residues among the four types of CNTs (Figure 1d). Most of the impurity elements are transition metals, such as Fe, Co, Mo, Y, Ni and $\mathrm{Cr}$, which is in agreement with previous report. ${ }^{16}$

In the following studies, the dynamic dissolution of metal residues from CNT-2 into artificial fluids was studied. The chemical compositions of the artificial fluids are listed in Table S1. Table S2 shows the metal composition and contents in CNT-2. There are trace amounts of $\mathrm{Mn}, \mathrm{Cu}, \mathrm{Zn}$ and $\mathrm{Co}$ together with the major metals $\mathrm{Fe}, \mathrm{Mo}, \mathrm{Cr}$ and $\mathrm{Ni}$.

\section{Mobilization of metal residues from CNTs into low-pH AGF}

The content of metals leached from $1.5 \mathrm{mg}$ of CNT-2 into $1 \mathrm{ml}$ of artificial fluid are shown in Figure 2a, and Figure $2 \mathrm{~b}$ shows the weight percentages of leached metal accounting for the total metal amount in CNT-2 at each indicated time point. In the low-pH AGF $(\mathrm{pH}=1.2)$, $\mathrm{Cu}$ and $\mathrm{Zn}$ had large mobilization rates $(\mathrm{Cu} 23.2 \%, \mathrm{Zn} \mathrm{33.7 \% )} \mathrm{after}$ $8 \mathrm{~h}$ of incubation. Except for $\mathrm{Mn}(2.2 \%)$ and $\mathrm{Fe}(1.3 \%)$, the mobilization rates of other metal species were no more than $1 \%$. The metal mobilization rate over extended incubation times varied widely. After 2 months of incubation, the mobilization rates of $\mathrm{Cu}$ and $\mathrm{Zn}$ reached $35 \%$ and $50 \%$, respectively, while that of the other metal species were $<10 \%$. The greater mobilization rates of $\mathrm{Cu}$ and $\mathrm{Zn}$ indicated that the bioavailability of these two metals is higher than that of the other metal species. After incubation for 6 months, the concentrations of metal mobilized from $1.5 \mathrm{mg}$ of CNTs into $1 \mathrm{ml}$ of artificial fluid were thus: Fe 3315, Ni 113, Mo 92, Cr 89, Cu 33, Zn 20, Mn, 9.5 Co, 0.71 (unit: p.p.b.). The addition of pepsin did not facilitate or inhibit metal mobilization.

Mobilization of metal residues from CNTs into mildly acidic ALF There was no leaching of Co or Mo from CNTs into mildly acidic ALF. For Cr, Mn, Fe and Ni, only extremely small quantities of metals leached from CNTs into ALF. It was clear that much more metal leached from CNTs into AGF $(\mathrm{pH}=1.2)$ than into ALF ( $\mathrm{pH}=4.5)$. This inverse relationship between $\mathrm{pH}$ and metal mobilization is expected because acid-soluble metals are more soluble in acidic solution. Unlike other metal species, the mobilization of $\mathrm{Cu}$ and $\mathrm{Zn}$ in ALF were close to and slightly lower than the data in AGF $(\mathrm{pH}=1.2)$. After $8 \mathrm{~h}$ of incubation, the mobilization of $\mathrm{Cu}$ and $\mathrm{Zn}$ had nearly reached a plateau (59\% and $69 \%$, respectively). The remarkable mobilization rates of $\mathrm{Cu}$ and $\mathrm{Zn}$ might be related to their high acid sensitivity as well as large degrees of bio-availability of $\mathrm{Cu}$ and $\mathrm{Zn}$. After 6 months of incubation, the concentrations of metal mobilized from $1.5 \mathrm{mg}$ of CNTs into $1 \mathrm{ml}$ of artificial fluid were thus: Fe 135, Ni 12, Cu 19, Zn 16, Cr 6, Mn 3 (unit: p.p.b.).

\section{Mobilization of metal residues from CNTs into nearly neutral artificial fluids}

In AIF ( $\mathrm{pH}$ 6.8) without trypsin, only Mo was released from CNTs in significant amounts (Figure $2 \mathrm{a}$ ). The addition of trypsin resulted in a slight enhancement of Ni mobilization and a greater enhancement for Mn mobilization. The addition of trypsin had no significant effect on Mo mobilization. The mobilization rates of Mo from CNTs into the AIF ( $\mathrm{pH}$ 6.8) were from $7 \%$ after $8 \mathrm{~h}$ of incubation to $13.5 \%$ after 2 months of incubation. There was a similar dissolution tendency in Gamble's solution (lung epithelial lining fluid stimulant), which was a 

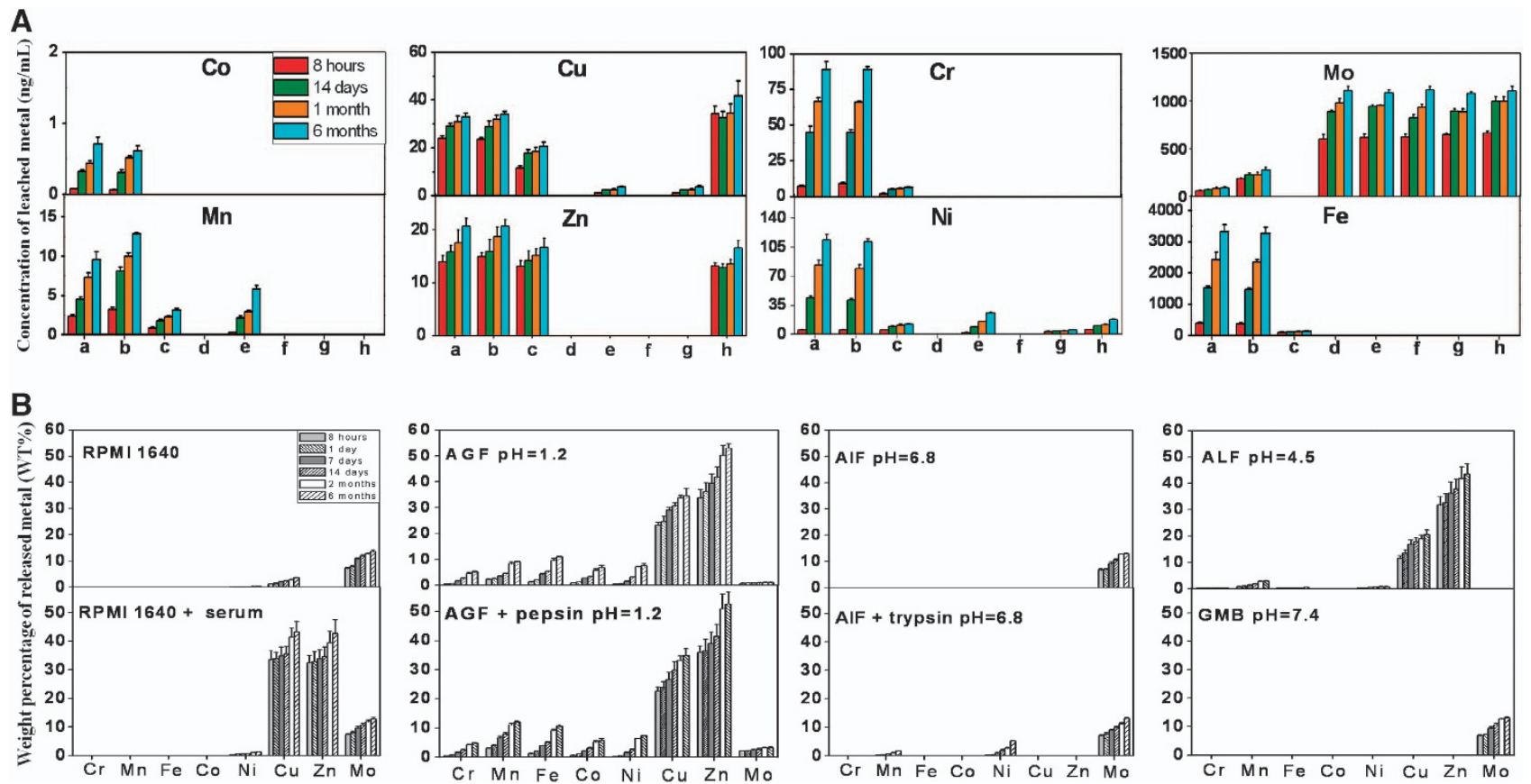

Figure 2 The mobilization of metal impurities from carbon nanotube (CNT)-2 into surrounding artificial fluids. (A) The concentrations of metal leached from $1.5 \mathrm{mg}$ of CNT-2 into $1 \mathrm{~g}$ of artificial fluid (unit, $\mathrm{ngg}^{-1}$ ). The labels on the horizontal axis represent various artificial fluids: a, artificial gastric fluid (AGF) pH 1.2; b, AGF + pepsin pH 1.2; c, artificial lysosomal fluid (ALF) pH 4.5; d, artificial intestinal fluid (AIF) pH 6.8; e, AlF + trypsin pH 6.8; f, GMB pH 7.4; g, RPMI 1640; and h, RPMI 1640 + serum. (B) The weight percentage of released metals accounting for the total metal content in CNT-2 at each indicated time point for each metal (wt \%). The y axis represents the weight percentage of released metal.

complex salt solution designed to mimic the salt balance of extracellular fluid.

\section{Mobilization of metal residues from CNTs into cell-free culture medium}

Only considerable quantities of Mo and extremely low quantities of $\mathrm{Ni}$ and $\mathrm{Cu}$ mobilized from CNTs into culture medium. The addition of serum generally enhanced metal mobilization, resulting in a dramatic enhancement of the mobilization of $\mathrm{Cu}$ and $\mathrm{Zn}$ and a slight enhancement of Ni mobilization. After incubation for $8 \mathrm{~h}$ in culture medium containing $10 \%$ serum, the mobilization rates of $\mathrm{Cu}$ and $\mathrm{Zn}$ reached $33.6 \%$ and $32.4 \%$, respectively, and the mobilization rate of $\mathrm{Cu}$ was even higher than that observed using AGF. The remarkable enhancing effects of serum on $\mathrm{Cu}$ and $\mathrm{Zn}$ mobilization could be attributed to the interaction of $\mathrm{Cu}$ and $\mathrm{Zn}$ with the proteins in the serum.

The experimental results suggest that the mobilization of metal residues from CNTs into biological microenvironments is a complex process that depends on the properties and constituents of the biological microenvironment, as well as the properties of the metal particles. Our results confirm that the $\mathrm{pH}$ and constituents (that is, protein) of biological fluids, the chemical properties of the metal particles (that is, acid sensitivity) and the incubation time affect metal mobilization. The low $\mathrm{pH}$ allows these metals to partition from the solid, encapsulated phase into solution, thereby yielding metallic species, and facilitates metal mobilization. The constituents of the biological microenvironment are complex, containing abundant proteins and inorganic salts. Proteins increase the dissolution rates of metal oxide particles through aqueous complexation (that is, aqueous species complex free ions released from the material's surface) and ligand-enhanced dissolution (that is, adsorbed natural organic material and organic acids, extracting surface metal atoms from NP surfaces). Therefore, metal dissolution is increased under acidic conditions as well as the presence of biological components, such as amino acids and peptides. In addition to the properties and constituents of the biological environment, the chemical property of the metal particles is also important to metal dissolution. In an acidic or wildly acidic physiological environment, acid-sensitive metal is prone to dissolve.

\section{ESR results for generation of free radicals}

Because metal residues could mobilize from CNTs into biological fluids, it is of interest whether mobilized metal could affect the generation of free radicals. Therefore, ESR spectroscopy was used to detect and quantify radicals generated by leachates, which were from the incubation of CNT-2 in artificial fluids for 2 months, and the three leachates were labeled as leachate-AIF, leachate-ALF and leachate-AGF. After incubating CNT-2 for 2 months, predominately 2838 p.p.b. of Fe lached into AGF, 121 p.p.b. of Fe into AIF and 1067 p.p.b. of Mo into ALF (Figure 3a).

In some cases, hydrogen peroxide was added to determine whether the leached metal could mediate a Fenton-like reaction, generating hydroxyl radicals. ESR signals were observed in leachate-AGF and leachate-ALF, but not in leachate-AIF, which indicated that a significant quantity of mobilized Mo could not mediate the generation of free radicals under the conditions of this experiment. The observed ESR spectra were characteristic of the hydroxyl radical (Figure 3b). The signal intensity from leachate-AGF was much higher than that from leachate-ALF; even after adding five times the concentration of $\mathrm{H}_{2} \mathrm{O}_{2}$, the ESR signal intensity generated by leachate-ALF was significantly lower than leachate-AGF. Acid leaching mobilizes metal residues in CNTs, allowing these metals to partition 


\begin{tabular}{lccccccc}
\hline & $\mathrm{Fe}$ & $\mathrm{Mo}$ & $\mathrm{Ni}$ & $\mathrm{Cr}$ & $\mathrm{Cu}$ & $\mathrm{Zn}$ & $\mathrm{Mn}$ \\
\hline leachate-AGF & $2838 \pm 269$ & $88 \pm 7$ & $105 \pm 2$ & $80 \pm 5$ & $31 \pm 2$ & $18 \pm 2$ & $8.9 \pm 0.7$ \\
leachate-ALF & $121 \pm 8$ & $\mathrm{ND}$ & $11 \pm 1$ & $5 \pm 0.3$ & $17 \pm 1$ & $15 \pm 2$ & $2.9 \pm 0.1$ \\
leachate-AIF & $\mathrm{ND}$ & $1067 \pm 37$ & $\mathrm{ND}$ & $\mathrm{ND}$ & $\mathrm{ND}$ & $\mathrm{ND}$ & $\mathrm{ND}$ \\
\hline
\end{tabular}

b

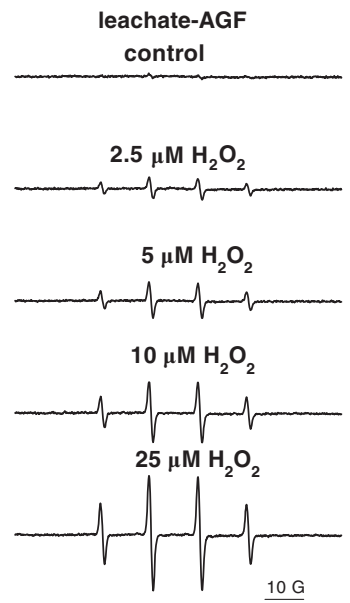

Figure $4 \mathrm{~b}$ shows the good positive correlation between the amount of radicals generated by CNTs at $\mathrm{pH} 1.2$ and the Fe content in CNTs. In summary, the observed dependence of the ESR signal on $\mathrm{pH}$, metal content and specific metal species indicates that the generation of hydroxyl radicals is mediated by acid-leachable metals instead of undissolved metal oxides or CNTs and that hydroxyl radicals were generated mainly by acid-leachable Fe rather than other metal species.

To more closely mimic the cellular milieu in these systems, biologically relevant reducing agents were added to create a more realistic model, improving the purely chemical Fenton-like reaction system used above. The reducing agents used were glutathione sulphydryl, cysteine (to mimic antioxidant enzymes containing a thiol in the active site), NADPH and NADH (to mimic biologically relevant reducing equivalents) and ascorbic acid (a classical antioxidant). These four types of CNTs were tested for their ability to generate radicals in a Fenton-like reaction. As shown in Figure 4c, CNTs were able to generate hydroxyl radicals under these experimental conditions. There were clear differences among the different CNTs tested. The amount of radicals generated appeared to be dependent on the $\mathrm{pH}$; a higher $\mathrm{pH}$ resulted in fewer observed radicals, which is consistent with our earlier results. Generally, samples that included reducing agents produced more radicals than those that did not. CNT-3, which had the highest metal content (in the form of iron), produced the most radicals. This trend was especially true at the low $\mathrm{pH}$ of 1.2 and time of $1 \mathrm{~min}$.

It was clear that adding $\mathrm{NADH}$ produced the highest level of

Figure 3 (a) The concentration level of metal leached from $1.5 \mathrm{mg}$ of carbon nanotube (CNT)-2 into $1 \mathrm{~g}$ of artificial fluid. Data are expressed as the mean \pm s.d. of three independent determinations $\left(\mathrm{ngg}^{-1}\right)$. (b) Hydroxyl radicals generated by leachate from CNT-2 incubated in artificial fluids for 2 months (leachate-AGF (artificial gastric fluid), leachate-ALF (artificial lysosomal fluid)). Samples contained $40 \mu \mathrm{l}$ of leachate, $5 \mu \mathrm{l}$ of $500 \mathrm{~mm}$ DMPO (5,5-dimethyl-pyrroline $\mathrm{N}$-oxide) and $5 \mu \mathrm{l}$ of solutions containing selected concentrations of $\mathrm{H}_{2} \mathrm{O}_{2}$. AlF, artificial intestinal fluid; ND, not detected.

from the solid, encapsulated phase into solution, yielding species that might affect the generation of free radicals.

To confirm this interpretation of the data, four types of CNTs having varying metal species and metal contents were dispersed in media with different pHs. As shown in Figure 4a, in a neutral environment ( $\mathrm{pH}$ 6.8), very weak ESR signals for the hydroxyl radical were observed. In the mildly acidic environment ( $\mathrm{pH} 4.0$ ), an ESR signal indicative of the hydroxyl radical could be observed for the CNT containing the highest level of Fe (CNT-3 9.3\% Fe), whereas no signal was detected in CNTs containing a low level of Fe (CNT-2 2\% $\mathrm{Fe}$ and CNT-1 $0.7 \% \mathrm{Fe}$ ) and pristine CNT-4 containing $11 \% \mathrm{Ni}$ and $3.3 \% \mathrm{Y}$. It was noted that $\mathrm{Fe}$, in contrast to other metal species, such as $\mathrm{Ni}$ and $\mathrm{Y}$, had a dominant role in mediating the generation of the hydroxyl radical. Furthermore, in an acidic environment ( $\mathrm{pH}$ 1.2), an ESR signal for hydroxyl radical could be found at varying intensity for all four CNTs. Comparing the ESR signals produced by CNTs in different $\mathrm{pHs}$ confirmed that a stronger ESR signal was produced in an environment of lower $\mathrm{pH}$. Therefore, this $\mathrm{pH}$-dependent response indicates that free radicals are produced by acid-leachable metals instead of undissolved metal oxides or CNTs.

Additionally, the observed ESR signal intensities were generally higher for CNTs having a higher Fe content, with the exception of CNT-4, which had no iron but showed higher signal intensity than both CNT-1 and CNT-2, suggesting a possible radical-generating role for Ni. No relationship was observed between levels of other metal species contained in CNTs and the level of hydroxyl radical generated.

radicals for all $\mathrm{pHs}$ and at all time points (Figure $4 \mathrm{c}$ and Supplementary Figure S1). Adding sodium ascorbate produced the ascorbyl radical, which was most pronounced at the high pH, 6.8 (not shown). The observed $\mathrm{pH}$ dependence of the radical generation might be due, at least in part, to the low solubility of metal ions, especially iron, at high $\mathrm{pH}$, and in part to the poor leaching of metals from the CNTs. This reasoning would also explain why the expected $\mathrm{pH}$-dependence of the ESR signal intensity was observed (that is, higher iron content results in more radical production). The results clearly indicate a role for reducing agents and antioxidants in the potential toxicities of these nanotubes. This effect might be due to the participation of reducing agents and antioxidants in redox cycling. The tendency of the reducing agents to participate in redox cycling appeared to be influenced by $\mathrm{pH}$. At the high $\mathrm{pH}$ (6.8), radical signal intensities were generally lower compared with the intensities at other pHs. The differences in signal intensities across the reducing agents might be partially explained by their different reducing potentials. Ascorbic acid was the reducing agent that produced the smallest change in signal intensities across $\mathrm{pHs}$ (with the exception of CNT-4), likely due to its strong radical scavenging activity.

It was evident that the roles of different metal species in generating free radicals were different. The participation of iron in the Fenton reaction is well established. Chromium and cobalt have also been shown to generate free radicals in Fenton-like reactions. ${ }^{32,33}$ However, it is not clear what role, if any, molybdenum and nickel might have in a Fenton-like reaction. Our results indicate that nickel can reduce hydrogen peroxide to produce hydroxyl radicals, as observed with CNT-4 (predominantly nickel, with a little cobalt, yttrium and no iron or chromium). Both nickel and cobalt are used industrially as catalysts in oxidation reactions. The role of each constituent metal in generating ROS is not clearly defined. The possible interaction between some of these constituents is even less understood. In spite of these uncertainties, we can conclude that, 
a
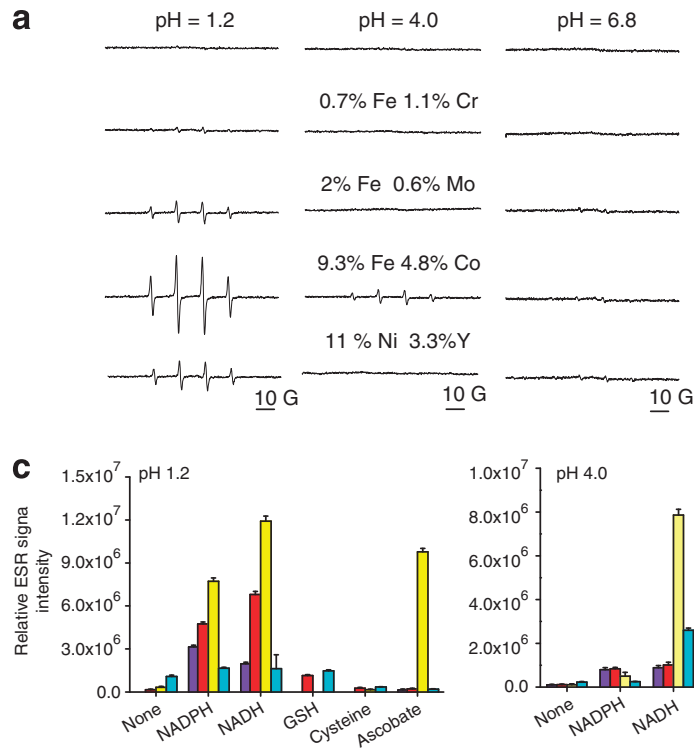

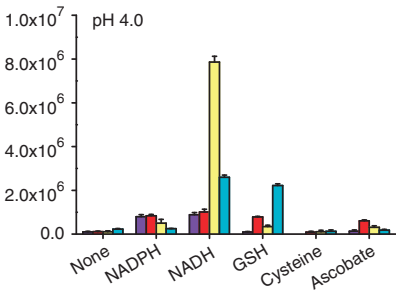

b
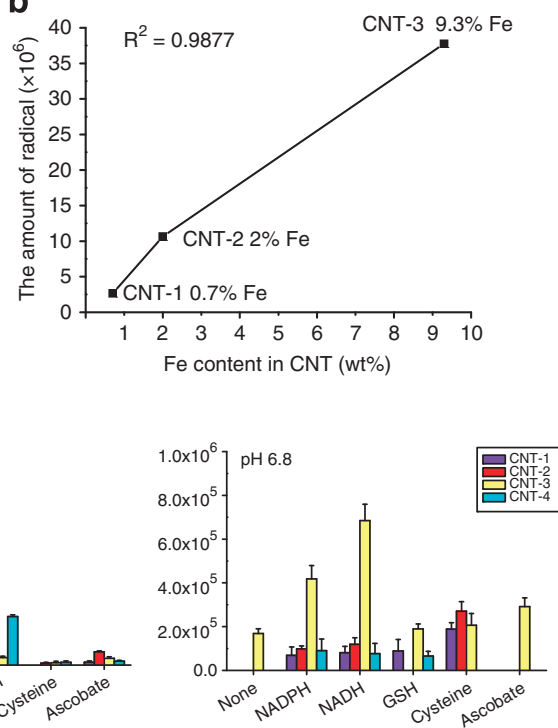

Figure 4 (a) Hydroxyl-radical formation from carbon nanotubes (CNTs) having different metal contents at different pHs. Samples contain $0.5 \mathrm{mg} m \mathrm{I}^{-1}$ of different single wall nanotubes, $0.1 \mathrm{~mm} \mathrm{H}_{2} \mathrm{O}_{2}$ and $50 \mathrm{~mm}$ 5,5-dimethyl-pyrroline $\mathrm{N}$-oxide (DMPO). Electron spin resonance (ESR) spectra were recorded 1 min after sample mixing. (b) The correlation between the amount of radicals from nanotubes and Fe content in CNTs. (c) Hydroxyl-radical formation from nanotubes having different metal contents at different pHs. Samples contain $0.5 \mathrm{mg} \mathrm{ml}^{-1}$ of different single wall nanotubes, $0.1 \mathrm{~mm} \mathrm{H}_{2} \mathrm{O}_{2}, 50 \mathrm{~mm}^{\mathrm{DMPO}}$ and $0.25 \mathrm{~mm}$ of various reducing agents. ESR spectra were recorded 1 min after sample mixing. The $\mathrm{x}$ axis represents different reducing agents, and the $\mathrm{y}$ axis represents the ESR signal intensity of the spin adduct (a.u.). GSH, reduced glutathione; NADH, reduced nicotinamide adenine dinucleotide; NADPH, reduced nicotinamide adenine dinucleotide phosphate.

under the experimental conditions described, these CNTs mediate the production of hydroxyl radicals, which might result in damage to biological targets.

\section{Cell viability, intracellular ROS and the possible underlying mechanism}

Free-radical generation associated with metals leached from CNTs might disturb the oxidation-reduction equilibrium of cells and induce oxidative stress and cytotoxicity. Therefore, we investigated the effects of CNTs and leached metal on cell viability. It has been reported that, after CNTs enter the blood system, there is rapid blood clearance and a rapid uptake and accumulation of CNTs in the reticuloendothelial system ${ }^{34}$ These accumulated CNTs and metals leaching out of the CNTs can be cleared by macrophages or enzymes. It is expected that as metals progressively leach from CNTs, the levels of ROS will increase and affect biological functions. To mimic the accumulation of leaching metals expected following in-vivo exposure to CNTs, pristine leachates were concentrated by freezing.

\section{Influence on cell viability}

So far, there has been no quantitative study about the respective effect of CNTs themselves and metal residues on cell viability. Therefore, CNTs with significantly different metal residues, including CNT-1 (2\% metal residues) and CNT-3 (15\% metal residues) were used. Compared with CNT-3, the low metal content in CNT-1 can be ignored, and CNT-1 can be regarded as standard samples of CNTs without metal residues. To make a semi-quantitative analysis, we make figures to show the correlation between cell viability and the concentration of CNTs as well as metal residues. As clearly shown in Figure 5a and Supplementary Figure S2, along with the increasing CNT concentration from 10 to $80 \mu \mathrm{g} \mathrm{ml}^{-1}$, the effect of the CNT standard (CNT-1) on cell viability was basically invariable and remained slightly higher than $80 \%$. By increasing the CNT-3 concentration from 8.5 to $76.5 \mu \mathrm{g} \mathrm{ml}^{-1}$, the absolute concentration of metal residues in CNT-3 increased from 1.5 to $13.5 \mu \mathrm{g} \mathrm{ml}^{-1}$. The cell viability presented a good negative correlation with the amount of metal residues in CNT-3 (Figure 5b). Therefore, it can be concluded that when the CNT concentration was below $80 \mu \mathrm{g} \mathrm{ml}^{-1}$, the metal residues had a dominant role in cytotoxicity and the effect of CNTs on cell viability was limited. When the CNT concentration exceeded $80 \mu \mathrm{g} \mathrm{ml}^{-1}$, CNTs (CNT-1) brought an obvious down effect on cell viability, which might be related to the aggregation of nanotubes. Our recent study have shown that quick binding of blood proteins to highpurity SWCNTs can greatly reduce the cytotoxicity, ${ }^{35}$ which can explain why we cannot observe cell death after exposure to CNTs below $80 \mu \mathrm{g} \mathrm{ml}^{-1}$.

As shown in Figure $5 c$, leachate-AGF with high Fe content induced the highest cytotoxicity, leachate-ALF with low Fe content induced intermediate levels of cytotoxicity and leachate-AIF without $\mathrm{Fe}$ induced the least cytotoxicity. The cytotoxicity depended on the concentration ratios and Fe content of the leachate. After cells were exposed to CNT-2, c-leachate and a mixture of both, exposure to c-leachate elicited a greater cytotoxity than exposure to CNT-2 (Figure 5d). An additive effect on cytotoxicity was observed when cells were exposed to both the leachate and CNT-2. Therefore, it may be concluded that CNT-induced cytotoxicity results from the co-effect of leached metal and CNTs themselves and that the leached metal has a major role.

\section{Measurement of intracellular ROS and SOD}

Our results are consistent with the dominant mechanism for toxicity being oxidative stress induced by bioavailable metals. For example, hydroxyl radicals can be generated through reactions that involve transition metal ions, such as the $\mathrm{Fe}^{2+}$ ion, via the Fenton reaction. The hydroxyl radical is highly reactive and can react with biological molecules to cause oxidative stress. 
a
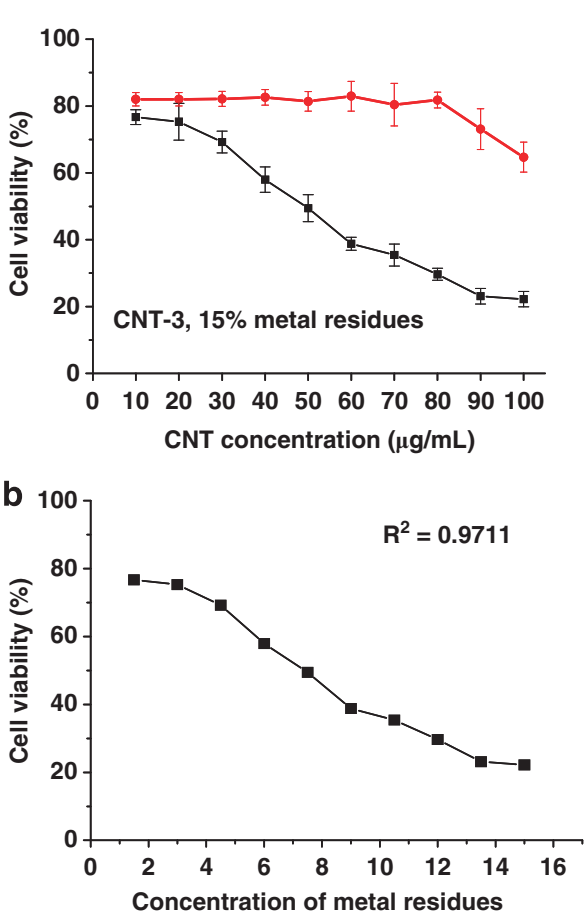
in CNT-3 $(\mu \mathrm{g} / \mathrm{mL})$

e Control

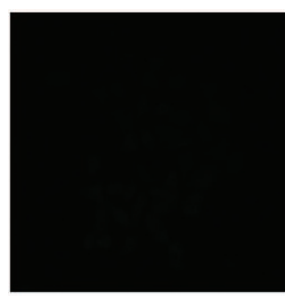

c-leachate

-ALF

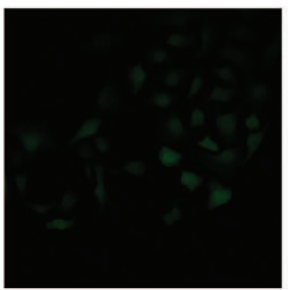

CNT-2
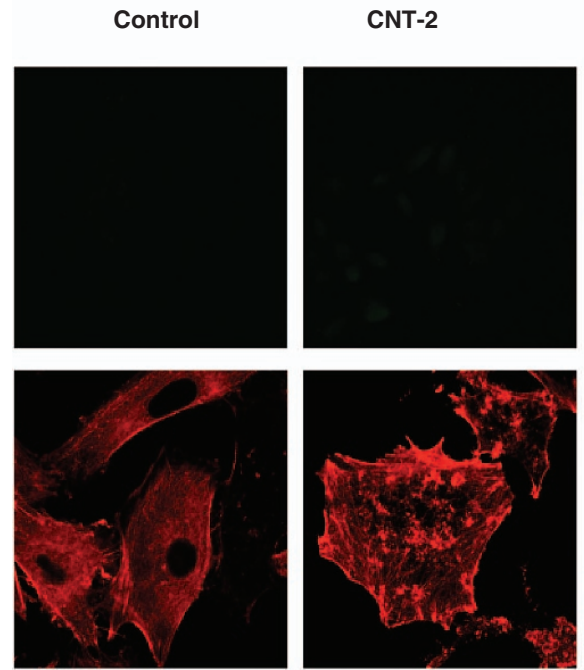

C
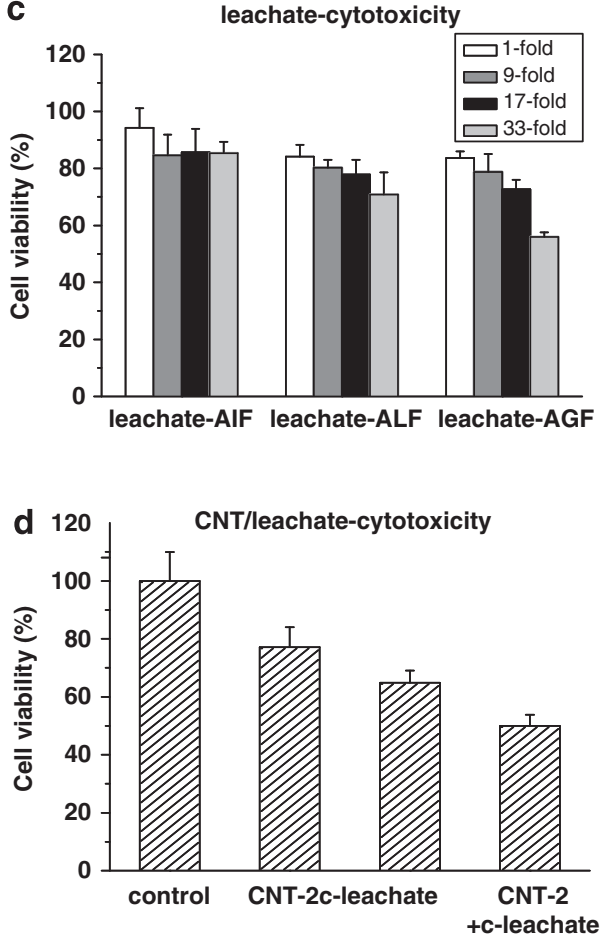

c-leachate

-AIF

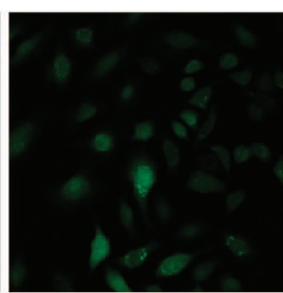

c-leachate

-AGF
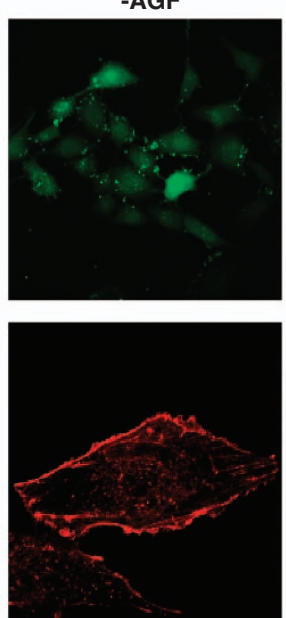

c-leachate

-AGF

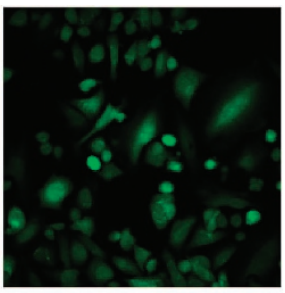

CNT-2+

c-leachate-AGF
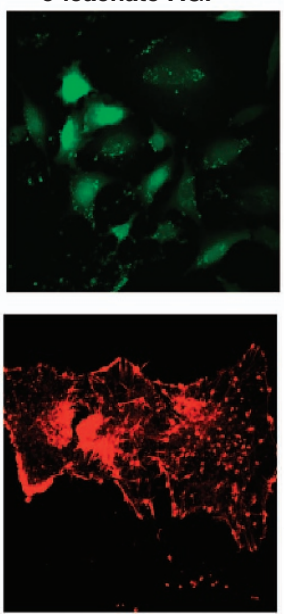

Figure 5 The measurement of viability and intracellular reactive oxidative species (ROS) of A549 cells. (a) The cell viability after treatment with carbon nanotube (CNT)-1 and CNT-3. Note: The $x$ axis represents the concentration of CNTs. (b) The correlation between cell viability and the content of metal residues in CNTs. (c) The cell viability after treatment with concentrated leachates (leachate-AIF (artificial intestinal fluid), leachate-ALF (artificial lysosomal fluid), leachate-AGF (artificial gastric fluid)). (d) The cell viability after treatment with $10 \mu \mathrm{g} \mathrm{ml} \mathrm{l}^{-1}$ of CNT-2, c-leachate (from CNT-2 incubation in AGF for 2 months) and a mixture of both. (e) Representative confocal microscope images showing the generation of intracellular ROS after respective treatment with concentrated leachates (c-leachate-ALF, c-leachate-AIF, c-leachate-AGF). (f) Representative confocal microscope images showing the generation of intracellular ROS and actin cytoskeleton organization after respective treatment for $24 \mathrm{~h}$ with $10 \mu \mathrm{g} \mathrm{ml} \mathrm{l}^{-1}$ of CNT-2, c-leachate-AGF and a mixture of both. 

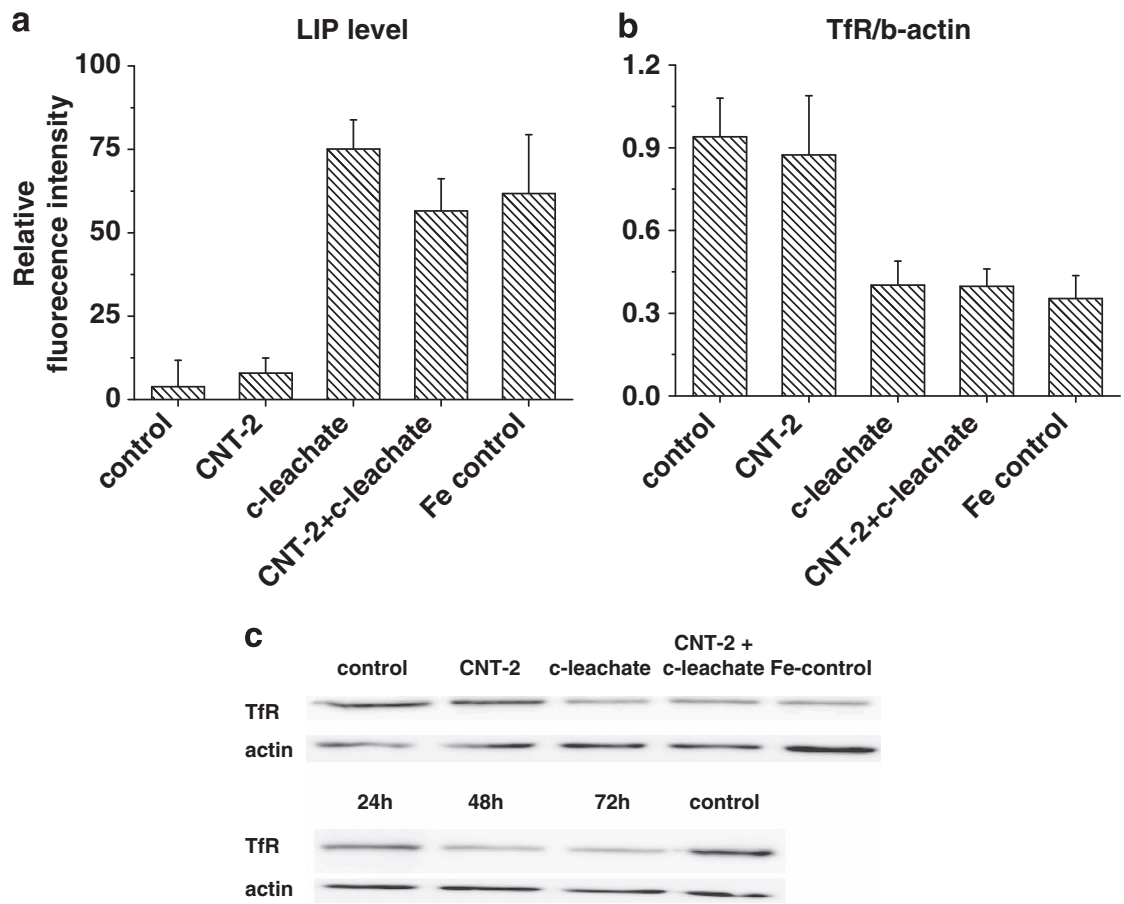

Figure 6 The assay of labile iron pool (LIP) and transferrin receptor (TfR). (a, b) The LIP level (a) and the expression levels of TfR protein (b) after respective treatment for $24 \mathrm{~h}$ with $10 \mu \mathrm{g} \mathrm{ml}{ }^{-1}$ of carbon nanotube (CNT)-2, c-leachate(artificial gastric fluid (AGF)), a mixture of both and iron control solution equal to the $\mathrm{Fe}$ content in c-leachate. (c) Western blot showing the expression levels of TfR protein and in parallel with b-actin expression as a loading control. In the upper lanes, cells were treated for $24 \mathrm{~h}$ with $10 \mu \mathrm{g} \mathrm{ml} \mathrm{l}^{-1}$ of CNT-2, c-leachate (AGF), mixture of both and a Fe control solution. In the lower lanes, cells were treated with c-leachate (AGF) for 24,48 and $72 \mathrm{~h}$.



Figure 7 Transport pathway, cellular and molecular mechanisms of toxicity associated with cellular exposure to carbon nanotubes and leached metal. Intracellular iron uptake can occur by transferrin receptor (TfR) on cellular membranes (the reductase present in acidified endosomes converts $\mathrm{Fe}^{3+}$ to $\mathrm{Fe}^{2+}$ before transport into the cytosolic space by divalent metal transporter-1 (DMT-1)) or carbon nanotube endocytosis/phagocytosis followed by acidenhanced $\mathrm{Fe}^{2+}$ release in endosome and lysosome. By increasing the intracellular iron, the level of labile iron pool (LIP) is upregulated. The resultant Fe ${ }^{2+}$ within the LIP (1) activates the regulatory iron regulatory protein/iron regulatory element (IRP/IRE) system; (2) is sequestered by chaperones or storage proteins, such as ferritin and metallothioneins (MTs); (3) reoxidizes into $\mathrm{Fe}^{3+}$ and effluxes out of the cell via CP (ceruloplasmin) and IREG1 (iron-regulated gene 1); or (4) participates in iron-catalyzed reactions to generate reactive species. SOD, superoxide dismutase. 
To test the connection between oxidative stress and cytotoxicity, the level of intracellular ROS was measured. The confocal image of intracellular DCF $\left(2^{\prime}, 7^{\prime}\right.$-dichlorofluorescin) fluorescence provided direct proof (Figure 5e). Consistent with the results of cell viability, concentrated leachate-AGF induced the greatest increase, concentrated leachate-ALF induced an intermediate increase and concentrated leachate-AIF induced a slight increase of intracellular ROS levels. Additionally, Supplementary Figure S3 provides a quantitative result of intracellular ROS level. Consistent with the preceding results, following treatment of cells with CNT-2 resulted in no detectable ROS, whereas treatment with c-leachate resulted in a dramatic increase in ROS. An additive effect on cytotoxicity was observed when cells were exposed to both the leachate and CNT-2 (Figure 5f). Additionally, in control culture medium A549, cells displayed firm or compact fibers, whereas in medium containing concentrated leachate, a disruption of the actin cytoskeleton was evident (Figure 5f). This result suggested a link between metal-mediated generation of ROS and the cytoskeleton. The cytoskeleton has a major role in the functions of cells, including adhesion, permeability and motility. In addition to maintaining cellular structural integrity through its threedimensional network of filamentous polymers, the cytoskeleton may participate in agonist-stimulated signal transduction.

ROS, formed as a natural byproduct of the normal metabolism of oxygen, have an important role in cell signaling. However, dramatically increased levels of ROS, arising from exposure to environmental pro-oxidants or from certain pathological conditions, can result in significant oxidative damage to cellular structures. Such dramatic increases in ROS elicit a condition commonly called oxidative stress. Excessive levels of ROS produced by metals leached from CNTs might overwhelm the protective effects of endogenous antioxidants, resulting in oxidative stress and damage to cells. To investigate whether metals leached from CNTs induce oxidative stress, we measured the activity of SOD, one type of major antioxidant enzyme in cells. As shown in Supplementary Figure S4 the activity of SOD was significantly reduced in cells treated with CNT-2 and is further reduced in cells treated with the c-leachate. For cells treated with both c-leachate and CNT, the effect on reduction of SOD activity appeared additive. The reduced activity of SOD provided indirect proof of cytotoxicity. The possible mechanism of cytotoxicity might be related to oxidative stress.

\section{Measurement of intracellular transferrin receptor (TfR) expression and labile iron pool (LIP) content}

Iron was the main metal residue contained in CNT-2, and the absolute mobilized amount of iron was significantly higher than that of the other metal residues. The preceding results indicate that acidsoluble iron has a critical role in the generation of hydroxyl radicals, the inhibition of cell viability and the promotion of intracellular ROS. Therefore, the level of the LIP was determined. The LIP comprises iron bound to low-affinity ligands that can vary in composition and quantity under different physiological conditions. The level of the LIP is regulated and maintained within a restricted range that meets the cell's requirements for iron but prevents excess free iron from triggering cellular damage. ${ }^{36-39}$ As shown in Figure 6a, compared with the control, the level of the LIP was upregulated after cells were treated with c-leachate, whereas treatment with CNT-2 had little effect on levels of the LIP. Upregulation of the LIP indicates that intracellular iron is more abundant and therefore could be available for ironcatalyzed reactions generating ROS and resulting in oxidative stress.

To maintain a balance of iron metabolism, the modified iron regulatory proteins (IRP) were no longer bonded to iron-responsive element (IRE), allowing TfR protein mRNA to be destroyed and allowing the higher expression of ferritin. TfR protein, whose expression is regulated by an IRE, is expressed on the membranes of cells and binds extracellular iron for transport into the cell. ${ }^{40}$ When intracellular levels of iron increase, the IRE/IRP system decreases expression of TfR protein on the membrane of the cells to reduce transport of iron into cells. TfR levels thus respond to the iron level in cells. Our experimental results showed that CNT-2 alone did not reduce the expression of TfR, and the reduction of TfR expression was mainly caused by c-leachate (Figure 6b). The western blot assay showed that downregulation of TfR protein expression was a timedependent response (Figure 6c). Additionally, due to several metal species contained in leachates, an iron control solution was used to confirm that the effects on the LIP level and TfR protein expression related to iron. The almost equal LIP level and TfR protein expression indicated that the observed upregulation of the LIP and the reduction of TfR expression mainly attributed to iron.

In summary, among several important metal residues in CNT-2, Fe had a major role as a component in generating free radicals, inhibiting cell viability and promoting intracellular ROS. The underlying mechanism was associated with intracellular iron metabolism. The upregulated LIP level indicated that intracellular iron was abundant, and the resultant Fe within LIP could participate in iron-catalyzed reactions to generate ROS. An excess of ROS induces oxidative stress, which reduces cell viability by eliciting oxidative damage to cellular targets, such as lipids, proteins and nucleic acids. To maintain iron homeostasis, the expression of TfR on cellular membranes is reduced, and the reduction of this iron carrier prevents more extracellular iron entering the cells, which will limit damage to cells. Finally, the transport pathway and molecular toxicity mechanism associated with cellular exposure to CNTs and leached metal are shown in Figure 7.

The results of the present study show the dynamic dissolution of metal residues from CNTs into biological microenvironments. The dissolution processes are complex and are influenced by the properties and constituents of the biological microenvironment, as well as the properties of the metal particles. Our results confirm that the $\mathrm{pH}$ and constituents (that is, proteins) of the biological microenvironment, the chemical properties of the metal particles (that is, acid sensitivity) and interaction time would affect the mobilization process.

Hydroxyl radicals are generated by acid-leachable metals rather than undissolved metal oxides or CNTs. A stronger ESR signal was produced in an environment of lower $\mathrm{pH}$, and the generation of free radical exhibited a good correlation with $\mathrm{Fe}$ content. Additionally, our results clearly indicate a role for reducing agents and antioxidants in the potential toxicities of these nanotubes. This effect might be due to their participation in redox cycling. The tendency of the reducing agents to participate in redox cycling appeared to be influenced by $\mathrm{pH}$. The differences in signal intensities across the reducing agents might be partially explained by their different reducing potentials. It was evident that the roles of different metal species in generating free radicals were different. The role of each constituent metal in generating ROS is not clearly defined. The possible interaction between some of these constituents is even less well-understood. Despite these uncertainties, we can conclude that, under the experimental conditions described, these CNTs mediate the production of hydroxyl radicals, which might result in damage to biological targets.

This study is the first to semi-quantitatively analyze the effect of CNTs and metal residues on cell viability. It was found that the metal residues had a dominant role in cytotoxicity, and the effect of CNTs on cell viability was limited. The cell viability presented an obvious 
negative correlation with the amount of metal residues in CNT. The reduction of cell viability related to abundant intracellular ROS. Excessive levels of ROS produced by metals leached from CNTs may overwhelm the protective effects of endogenous antioxidants, resulting in oxidative stress and damage to cells, which is highly dependent on the released or dissolved ability of metal residues in different biologically simulated fluids.

\section{CONFLICT OF INTEREST}

The authors declare no conflict of interest.

\section{ACKNOWLEDGEMENTS}

This work was financially supported by the National Basic Research Programs in the Ministry of Science and Technology of China (2011CB933401, 2012CB934000) and the Natural Science Foundation of China (10975040, 21277037) and the Danish Agency for Science Technology and Innovation (09067185). This work was partially supported by a regulatory science grant under the FY11 FDA Nanotechnology CORES Program (JY). We gratefully acknowledge the comments of Wayne Wamer (Center for Food Safety and Applied Nutrition/US Food and Drug Administration) during the preparation of this manuscript. This article is not an official US Food and Drug Administration (FDA) guidance or policy statement. No official support or endorsement by the US FDA is intended or should be inferred.

1 Colvin, V. L. The potential environmental impact of engineered nanomaterials. Nat Biotechnol. 21, 1166-1170 (2003).

2 Nel, A. E., Mädler, L., Velegol, D., Xia, T., Hoek, E. M. V., Somasundaran, P., Klaessig, F., Castranova, V. \& Thompson, M. Understanding biophysicochemical interactions at the nano-bio interface. Nat. Mater. 8, 543-557 (2009).

3 Zhao, Y., Xing, G. \& Chai, Z. Nanotoxicology: are carbon nanotubes safe? Nat. Nanotechnol. 3, 191-192 (2008).

4 Liu, Y., Zhao, Y., Sun, B. \& Chen, C. Understanding the toxicity of carbon nanotubes. Acc. Chem. Res. (e-pub ahead of print 21 September 2012; doi: 10.1021/ar300028m).

5 Krauss, T. D. Biosensors: nanotubes light up cells. Nat. Nanotechnol. 4, 85-86 (2009).

6 Kam, N. W. S., Jessop, T. C., Wender, P. A. \& Dai, H. Nanotube molecular transporters: internalization of carbon nanotube-protein conjugates into mammalian cells. J. Am. Chem. Soc. 126, 6850-6851 (2004).

7 Liu, B., Li, X., Li, B., Xu, B. \& Zhao, Y. Carbon nanotube based artificial water channel protein: membrane perturbation and water transportation. Nano Lett. 9, 1386-1394 (2009).

8 Liu, Z., Cai, W., He, L., Nakayama, N., Chen, K., Sun, X., Chen, X. \& Dai, H. In vivo biodistribution and highly efficient tumour targeting of carbon nanotubes in mice. Nat. Nanotechnol 2, 47-52 (2006).

9 Wang, L., Liu, Y., Li, W., Jiang, X., Ji, Y., Wu, X., Xu, L., Qiu, Y., Zhao, K. \& Wei, T. Selective targeting of gold nanorods at the mitochondria of cancer cells: implications for cancer therapy. Nano Lett. 11, 772-780 (2011).

10 De La Zerda, A., Zavaleta, C., Keren, S., Vaithilingam, S., Bodapati, S., Liu, Z., Levi, J., Smith, B. R., Ma, T. J. \& Oralkan, O. Carbon nanotubes as photoacoustic molecular imaging agents in living mice. Nat. Nanotechnol 3, 557-562 (2008).

11 Takagi, D., Homma, Y., Hibino, H., Suzuki, S. \& Kobayashi, Y. Single-walled carbon nanotube growth from highly activated metal nanoparticles. Nano Lett. 6, 2642-2645 (2006).

$12 \mathrm{Hu}, \mathrm{H}$., Zhao, B., Itkis, M. E. \& Haddon, R. C. Nitric acid purification of single-walled carbon nanotubes. J. Phys. Chem. B 107, 13838-13842 (2003).

13 Park, T. J., Banerjee, S., Hemraj-Benny, T. \& Wong, S. S. Purification strategies and purity visualization techniques for single-walled carbon nanotubes. J. Mater. Chem. 16, 141-154 (2006).

14 Chen, X., Chen, C., Chen, Q., Cheng, F., Zhang, G. \& Chen, Z. Non-destructive purification of multi-walled carbon nanotubes produced by catalyzed CVD. Mater. Lett. 57, 734-738 (2002)

15 Hou, P., Bai, S., Yang, Q., Liu, C. \& Cheng, H. Multi-step purification of carbon nanotubes. Carbon N Y 40, 81-85 (2002).

16 Ge, C., Li, W., Li, Y., Li, B., Du, J., Qiu, Y., Liu, Y., Gao, Y., Chai, Z. \& Chen, C. Significance and systematic analysis of metallic impurities of carbon nanotubes produced by different manufacturers. J. Nanosci. Nanotechnol. 11, 2389-2397 (2011).

17 Choi, J. H., Nguyen, F. T., Barone, P. W., Heller, D. A., Moll, A. E., Patel, D., Boppart, S. A. \& Strano, M. S. Multimodal biomedical imaging with asymmetric single-walled carbon nanotube/iron oxide nanoparticle complexes. Nano Lett. 7, 861-867 (2007).
18 Al Faraj, A., Cieslar, K., Lacroix, G., Gaillard, S., Canet-Soulas, E. \& Crémillieux, Y. In vivo imaging of carbon nanotube biodistribution using magnetic resonance imaging. Nano Lett. 9, 1023-1027 (2009).

19 Wang, C., Ma, X., Ye, S., Cheng, L., Yang, K., Guo, L., Li, C., Li, Y. \& Liu, Z. Protamine functionalized single-walled carbon nanotubes for stem cell labeling and in vivo Raman/magnetic resonance/photoacoustic triple-modal imaging. Adv. Funct. Mater. 22, 2363-2375 (2012)

20 Isobe, H., Tanaka, T., Maeda, R., Noiri, E., Solin, N., Yudasaka, M., lijima, S. \& Nakamura, E. Preparation, purification, characterization, and cytotoxicity assessment of water-soluble, transition-metal-free carbon nanotube aggregates. Angew. Chem. Int. Ed 45, 6676-6680 (2006).

21 Pulskamp, K., Diabaté, S. \& Krug, H. F. Carbon nanotubes show no sign of acute toxicity but induce intracellular reactive oxygen species in dependence on contaminants. Toxicol. Lett. 168, 58-74 (2007).

22 Kagan, V., Tyurina, Y., Tyurin, V., Konduru, N., Potapovich, A., Osipov, A., Kisin, E., Schwegler-Berry, D., Mercer, R. \& Castranova, V. Direct and indirect effects of single walled carbon nanotubes on RAW 264.7 macrophages: role of iron. Toxicol. Lett. 165, 88-100 (2006).

23 Liu, X., Gurel, V., Morris, D., Murray, D. W., Zhitkovich, A., Kane, A. B. \& Hurt, R. H. Bioavailability of nickel in single-wall carbon nanotubes. Adv. Mater. 19, 2790-2796 (2007)

24 Ge, C., Meng, L., Xu, L., Bai, R., Du, J., Zhang, L., Li, Y., Chang, Y., Zhao, Y. \& Chen, C. Acute pulmonary and moderate cardiovascular responses of spontaneously hypertensive rats after exposure to single-wall carbon nanotubes. Nanotoxicology 6, 526-542 (2011).

25 Guo, L., Morris, D. G., Liu, X., Vaslet, C., Hurt, R. H. \& Kane, A. B. Iron bioavailability and redox activity in diverse carbon nanotube samples. Chem. Mater. 19, 3472-3478 (2007).

26 Zhao, F., Chen, C., Liu, Y., Chang, X. \& Zhao, Y. Cellular uptake, intracellular trafficking, and cytotoxicity of nanomaterials. Small 7, 1322-1337 (2011).

27 Xia, T., Li, N. \& Nel, A. E. Potential health impact of nanoparticles. Annu. Rev. Public Health 30, 137-150 (2009).

$28 \mathrm{Li}, \mathrm{Y}$. F. \& Chen, C. Fate and toxicity of metallic and metal-containing nanoparticles for biomedical applications. Small 7, 2965-2980 (2011).

$29 \mathrm{Nel}$, A., Xia, T., Mädler, L. \& Li, N. Toxic potential of materials at the nanolevel. Science 311, 622-627 (2006)

30 Shvedova, A. A., Kisin, E., Murray, A. R., Johnson, V. J., Gorelik, O., Arepalli, S. Hubbs, A. F., Mercer, R. R., Keohavong, P. \& Sussman, N. Inhalation vs. aspiration of single-walled carbon nanotubes in C57BL/6 mice: inflammation, fibrosis, oxidative stress, and mutagenesis. Am. J. Physiol. Lung Cell. Mol. Physiol 295, L552-L565 (2008)

31 Ge, C., Lao, F., Li, W., Li, Y., Chen, C., Qiu, Y., Mao, X., Li, B., Chai, Z. \& Zhao, Y. Quantitative analysis of metal impurities in carbon nanotubes: efficacy of different pretreatment protocols for ICPMS spectroscopy. Anal. Chem. 80, 9426-9434 (2008).

32 Leonard, S.M, Gannett, P, Rojanasakul, Y., Schwegler-Berry, D., Castranova, V. Vallyathan, V. \& Shi, X. Cobalt-mediated generation of reactive oxygen species and its possible mechanism. J. Inorg. Biochem. 70, 239-244 (1998).

33 Borthiry, G. R., Antholine, W. E., Kalyanaraman, B., Myers, J. M. \& Myers, C. R. Reduction of hexavalent chromium by human cytochrome b5: Generation of hydroxyl radical and superoxide. Free Rad. Biol. Med 42, 738-755 (2007).

34 Yang, S. T., Wang, X., Jia, G., Gu, Y., Wang, T., Nie, H., Ge, C., Wang, H. \& Liu, Y. Long term accumulation and low toxicity of single-walled carbon nanotubes in intravenously exposed mice. Toxicol. Lett. 181, 182-189 (2008)

35 Ge, C., Du, J., Zhao, L., Wang, L., Liu, Y., Li, D., Yang, Y., Zhou, R., Zhao, Y. \& Chai, Z. Binding of blood proteins to carbon nanotubes reduces cytotoxicity. Proc. Natl. Acad. Sci. USA 108, 16968-16973 (2011).

36 Kruszewski, M. Labile iron pool: the main determinant of cellular response to oxidative stress. Mutation Res./Fund. Mol. Mech. Mutagenesis 531, 81-92 (2003).

37 Prus, E. \& Fibach, E.. Flow cytometry measurement of the labile iron pool in human hematopoietic cells. Cytometry Part A 73, 22-27 (2008)

38 Kakhlon, O., Gruenbaum, Y. \& Cabantchik, Z. I. Repression of the heavy ferritin chain increases the labile iron pool of human K562 cells. Biochem. J 356, 311-316 (2001)

39 Muckenthaler, M. U., Galy, B. \& Hentze, M. W. Systemic iron homeostasis and the ironresponsive element/iron-regulatory protein (IRE/IRP) regulatory network. Annu. Rev. Nutr. 28, 197-213 (2008).

40 Roy, C. N., Blemings, K. P., Deck, K. M., Davies, P. S., Anderson, E. L., Eisenstein, R. S. \& Enns, C. A. Increased IRP1 and IRP2 RNA binding activity accompanies a reduction of the labile iron pool in HFE-expressing cells. J. Cell. Physiol. 190, 218-226 (2002)

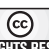

This work is licensed under the Creative Commons Attribution-NonCommercial-No Derivative Works 3.0 Unported License. To view a copy of this license, visit http://creativecommons.org/licenses/by-nc-nd/3.0/

Supplementary Information accompanies the paper on the NPG Asia Materials website (http://www.nature.com/am) 